\title{
Rs41291957 polymorphism in the promoter region of microRNA-143 serves as a prognostic biomarker for patients with intracranial hemorrhage
}

\author{
XIAOBO YANG ${ }^{1}$, ZONGDUO GUO $^{1}$, FANG CAO $^{2}$, ZHIPENG TENG $^{3}$, ZHIJIAN HUANG $^{1}$ and XIAOCHUAN SUN ${ }^{1}$ \\ ${ }^{1}$ Department of Neurosurgery, The First Affiliated Hospital of Chongqing Medical University, \\ Chongqing 400010; ${ }^{2}$ Department of Cerebrovascular Disease, The First Affiliated Hospital of Zunyi Medical College, \\ Zunyi, Guizhou 563000; ${ }^{3}$ Chongqing Traditional Chinese Medicine Hospital, Chongqing 400000, P.R. China
}

Received May 28, 2018; Accepted November 6, 2020

DOI: $10.3892 / \mathrm{mmr} .2021 .11928$

\begin{abstract}
The present study aimed to investigate the function of the single nucleotide polymorphism (SNP) rs41291957 in the prognosis of intracerebral hemorrhage ( $\mathrm{ICH})$. In addition, the molecular mechanisms underlying the role of microRNA (miR)-143, Toll-like receptor 2 (TLR2) and interleukin-16 (IL-16) were studied in patients with ICH that carried different alleles in the locus of the rs41291957 SNP. Kaplan-Meier survival curves were calculated for 182 patients with $\mathrm{ICH}$, genotyped as $\mathrm{CC}$, presenting a cytosine in both chromosome, CT, presenting both variants, and TT, presents a thymine in both chromosomes. In addition, the possible regulatory relationships between miR-143 and TLR2/IL-16 were studied using computational analysis, luciferase assays and western blot assay. In addition, the inflammatory profiles of cerebrospinal fluid (CSF) and serum samples collected from the subjects were compared. The patients genotyped as TT presented the lowest survival rate, while patients genotyped as CC presented the highest survival rate. TLR2 mRNA was identified as a potential target of miR-143, while IL-16 showed no direct interaction with miR-143. The above regulatory relationships were further investigated using cells transfected with miR-143 precursor or TLR2 small interfering RNA. In addition, the expression levels of inflammatory factors, such as tumor necrosis factor $\alpha$, interferon, IL-6, IL-10 and NF-L-6, were highest in the CSF/serum samples collected from patients genotyped as TT and lowest in patients genotyped as CC. By contrast, the expression levels of miR-143 showed an opposite trend in the expression of the above inflammatory factors. The rs41291957 SNP, located in the promoter region of miR-143,
\end{abstract}

Correspondence to: Dr Xiaochuan Sun, Department of Neurosurgery, The First Affiliated Hospital of Chongqing Medical University, 1 Youyi Road, Chongqing 400010, P.R. China

E-mail: iaichsah@yeah.net

Key words: intracranial haemorrhage, microRNA-143, Toll-like receptor 2, inflammation, rs4129195 reduced the expression of miR-143 and upregulated the expression of the pro-inflammatory factor TLR2, eventually leading to a poorer prognosis in patients with $\mathrm{ICH}$.

\section{Introduction}

Intracerebral hemorrhage ( $\mathrm{ICH})$, a primary hemorrhage in the parenchymal region, is a debilitating disease causing damage to the blood-brain barrier (BBB) and cerebral edema (1-3). ICH not only damages surrounding brain tissues, but can also cause secondary complications involving pro-inflammatory reactions, such as the infiltration of inflammatory cells into the central nervous system and stimulation of microglial cells (4-6).

Upon stimulation of microglial cells and astrocytes, the inflammatory response leads to the damage of BBB and the onset of edema in the brain (6). Subsequently, leukocytes are recruited to the site of injury, leading to the activation of pro-inflammatory mediators that can be potentially toxic to neurons (3-7). Synergistic effects of these events eventually lead to massive death of neuronal cells and further neurological damages (7). ICH causes both chronic and acute damages to brain tissues, through primary mechanical injury caused by hematomas and secondary complications induced by inflammatory mediators (8). The management of neuronal damage after ICH onset relies on the prevention of secondary brain damage (9).

MicroRNAs (miRNAs) are short (18-25 nucleotides in length) non-coding RNA transcripts evolutionarily conserved in eukaryotic cells, and are essential in gene regulation (10). By binding to the 3'-untranslated regions (3'-UTR) of their target genes, miRNAs can suppress gene expression through translational inhibition or mRNA degradation (11). In addition, miRNAs play a critical role in tumorigenesis. miRNAs(miR)-145 and miR-143, located on chromosome 5 in humans, are downregulated in colon cancer (12). A previous study showed that silencing of miR-143 is implicated in the initiation of immune responses (13). In addition, a previous study demonstrated that the expression of miR-143 is suppressed in colonic cancer (14). Moreover, miR-143 dysregulation is essential for the induction of inflammation in colon cancer (12-14). 
A single nucleotide polymorphism (SNP), rs41291957, in the sequence of pre-miR-143 has been shown to alter the transcription and expression of miR-143, thereby participating in the process of tumorigenesis (15). However, further studies are required to understand the effect of this SNP on miR-143 expression. Since miR-143 is part of a bicistronic cluster of miRNA genes, the SNPs in the promoter of miR-143 were analyzed and identified, including rs4705341, rs353293, rs353292 and rs41291957. These SNPs were revealed to be significantly associated with an increased risk of colorectal cancer $(15,16)$. By contrast, the SNPs rs3733846, rs3733845, rs17796757 and rs4705343 played a protective role against the pathogenesis of colorectal cancer (17). SNP rs41291957 is located in the promoter region of miR-143 and can reduce the transcription efficiency of the miR-143 promoter, thereby decreasing the expression of miR-143 (18). Toll-like receptor 2 (TLR2) was found to be a direct target of miR-143 (19). Furthermore, TLR2 expression is associated with the severity of inflammation and may affect the prognosis of patients with ICH $(20,21)$. The present study investigated the association between SNP rs41291957 and the prognosis of patients with ICH.

\section{Materials and methods}

Human subjects and sample collection. A total of 182 patients with ICH (age, 44-77 years; 109 male patients and 73 female patients) were enrolled in the present study between September 2015 and August 2017. Serum and CSF samples were collected from all patients. The patients were divided into two groups based on the prognosis: i) 150 patients with $\mathrm{ICH}$ were discharged from the hospital after treatment and were allocated to the group of survived patients; and ii) 32 patients with $\mathrm{ICH}$, who died in the hospital, were allocated to the group of deceased patients. In addition, the 182 patients were also divided into three groups based on rs41291957 SNP genotype: i) $\mathrm{CC}$, presenting a cytosine in both chromosome $(n=28)$, ii) CT, presenting both variants $(n=67)$ and iii) TT, presents a thymine in both chromosomes $(n=87)$. All patients with ICH were diagnosed by pathological examination. The study was approved by The First Affiliated Hospital of Chongqing Medical University Ethics Committee. All patients and their families had given the informed consent.

SNP sequencing. The rs41291957 SNP genotypes in the 182 patients were determined by PCR and denaturing high performance liquid chromatography (DHPLC) using the serum samples collected from each patient. The primers used for PCR amplification with Pfu DNA polymerase (Promega Corporation) were designed using Primer Premier 5 Oligo $^{\text {TM }} 6$ software (www.premierbiosoft.com/primerdesign). The primer sequences were as follows: Allele $C$ forward, 5'-AAT TACAACAGCCTCTCGG-3'; allele T forward, 5'-GAATTA CAACAGCCTCTTGG-3' and allele $\mathrm{C} / \mathrm{T}$ reverse, 5'-GCA CTGCACCTCAGGC-3'. The thermocycling conditions included an initial denaturation at $94^{\circ} \mathrm{C}$ for $2 \mathrm{~min}$, followed by 35 cycles of $94^{\circ} \mathrm{C}$ for $1 \mathrm{~min}, 52^{\circ} \mathrm{C}$ for $1 \mathrm{~min}$, and $72^{\circ} \mathrm{C}$ for $1 \mathrm{~min}$. Subsequently, PCR products $(50 \mu \mathrm{l})$ were extended for $5 \mathrm{~min}$ at $72^{\circ} \mathrm{C}$, and the genotypes of rs41291957 SNP were measured by DHPLC using the WAVE ${ }^{\mathrm{TM}}$ DNA Fragment Analysis system (ADS Biotec Ltd.) under partially denaturing conditions. The column (4.6x250 mm; $5 \mu \mathrm{m}$; ADS Biotec Ltd.) was maintained at $59.3^{\circ} \mathrm{C}$ and the mobile phase (water and methanol) flow rate was $0.9 \mathrm{ml} / \mathrm{min}$.

$R N A$ isolation and reverse transcription-quantitative (RT-qPCR). The expression levels of miR-143, TLR2 mRNA and interleukin-16 (IL-16) mRNA in clinical samples and cultured cells were measured by RT-qPCR. Total RNA of the samples was extracted using a TRIzol kit, according to the manufacturer's instructions (Invitrogen, Thermo Fisher Scientific, Inc.). RNA concentration was calculated using the 260/280 absorbance ratio. Reverse transcription to cDNA was carried out using an RT kit (Promega Corporation) according to the manufacturer's protocol. The primers for the PCR reactions were designed using Primer 5.0 software (www.premierbiosoft.com/primerdesign) according to the gene sequences of miR-143, TLR2 mRNA and IL-16 mRNA obtained from the GenBank database (db.cngb.org). The following primer pairs were used for qPCR: miR-143 forward, 5'-GCAGTGCTGCATCTCTG-3' and reverse, 5'-GAACATGTCTGCGTATCTC-3'; TLR2 forward, 5'-CTT CACTCAGGAGCAGCAAGCA-3' and reverse, 5'-ACACCA GTGCTGTCCTGTGACA-3'; IL-16 forward, 5'-TTGGAC ACAGGGTTCTCGCTCA-3' and reverse, 5'-AGCAGGGAG ATAACGGACTGAC-3'; and U6 forward, 5'-TTATGGGTC CTAGCCTGAC-3' and reverse, 5'-CACTATTGCGGGTCT GC-3'. The total volume of the RT-qPCR reaction system was $20 \mu \mathrm{l}$, which contained $10 \mu \mathrm{l}$ SYBR Premix Ex Taq (Takara Bio Inc.), $0.8 \mu \mathrm{l}$ forward primer, $0.8 \mu \mathrm{l}$ reverse primer, $0.4 \mu 1$ ROX reference dye II (Invitrogen; Thermo Fisher Scientific, Inc.), $2 \mu 1$ DNA templates and $6 \mu 1$ distilled water. The thermocycling conditions were as follows: Initial denaturation at $95^{\circ} \mathrm{C}$ for $30 \mathrm{sec}$, followed by 40 cycles of $95^{\circ} \mathrm{C}$ for $5 \mathrm{sec}$ and $60^{\circ} \mathrm{C}$ for $30 \mathrm{sec}$. The PCR results were verified by the melting curve using actin as a reference control. The relative level of target gene expression was quantified using the $2^{-\Delta \Delta \mathrm{Cq}}$ method (22) and normalized to the internal reference gene U6.

Cell culture and transfection. THP-1 (human monocytic cells) and human pulmonary artery smooth muscle cells (HPASMC) obtained from American Type Culture Collection were cultured in DMEM (Gibco; Thermo Fisher Scientific, Inc.) supplemented with $10 \%$ fetal bovine serum (Invitrogen; Thermo Fisher Scientific, Inc.). At a confluence rate of 80-90\%, the cells were trypsinized with a $0.25 \%$ EDTA-trypsin solution, precipitated by centrifugation at $167.7 \mathrm{x} g$ for $15 \mathrm{~min}$ at $4^{\circ} \mathrm{C}$, resuspended and seeded into 24 -well plates at a density of $4 \times 10^{5}$ cells per well. After an overnight incubation at $37^{\circ} \mathrm{C}$ and $5 \% \mathrm{CO}_{2}$, the cells were transfected with $50 \mu \mathrm{M}$ miR-143 precursor (5'-UGAGAUGAAGCACUGUAGCUC-3'), TLR2 siRNA (5'-GGAUCCUCGUGGAUAUCAAUU-3'), IL-16 SiRNA (5'-TCACCTCAACTCCAGTCCGTA-3') or miRNA scramble negative controls (5'-UGGGCGUAUAGACGUGUU ACAC-3'; all purchased from Santa Cruz Biotechnology, Inc.) using Lipofectamine ${ }^{\circledR} 2000$ (Invitrogen; Thermo Fisher Scientific, Inc.), according to the manufacturer's instructions. After $48 \mathrm{~h}$ of cell transfection at $4^{\circ} \mathrm{C}$, the cells were collected for subsequent experimentation. Each transfection experiment was repeated $\geq 3$ times. 
Luciferase assay. To investigate the regulatory relationship between miR-143 and IL-16, and between miR-143 and TLR2, TargetScan (version 7.2; (targetscan.org) bioinformatics software was used to predict the binding sites of miR-143 in IL-16 and TLR2. The 3'UTRs of IL-16 and TLR2 containing the binding sites of miR-143 were amplified by PCR and cloned into pGL3 vectors (Promega Corporation). The amplification was performed on a PTC-100 thermocycler (Bio-Rad Laboratories, Inc.) using the following thermocycling conditions: $95^{\circ} \mathrm{C}$ for $30 \mathrm{sec}, 40$ cycles of $30 \mathrm{sec}$ at $95^{\circ} \mathrm{C}, 2 \mathrm{~min}$ at $58^{\circ} \mathrm{C}$ and $30 \mathrm{sec}$ at $68^{\circ} \mathrm{C}, 72^{\circ} \mathrm{C}$ for $5 \mathrm{~min}$. Taq DNA polymerase (Sigma-Aldrich; Merck $\mathrm{KGaA}$ ) was used for PCR. In addition, site-directed mutagenesis was carried out in the putative binding sites of miR-143 in the 3'UTRs of IL-16 (forward, 5'-TTGGACACA GGGTTCTCGCTCA-3' and reverse, 5'-AGCAGGGAGATA ACGGACTGAC-3') and TLR2 (forward, 5'-CTTCACTCA GGAGCAGCAAGCA-3' and reverse, 5'-ACACCAGTGCTG TCCTGTGACA-3'), and the mutated sequences were also amplified by PCR and cloned into pcDNA3.1 vectors (Promega Corporation) according to the aforementioned protocol. THP-1 and HPASMC cells were co-transfected with miR-143 mimic and plasmids carrying the wild-type or mutant IL-16 or TLR2 using Lipofectamine ${ }^{\circledR} 2000$ (Invitrogen; Thermo Fisher Scientific, Inc.). After $48 \mathrm{~h}$ of transfection, cells were harvested and the luciferase activity of transfected cells was measured by a dual luciferase reporter assay system (Promega Corporation) on a luminometer (TD20/20; Turner Designs) at $4^{\circ} \mathrm{C}$. Firefly luciferase activity was obtained and normalized to the value of Renilla luciferase activity. Each experiment was repeated $\geq 3$ times.

Western blot analysis. Total protein was extracted using a urea buffer containing a protease inhibitor cocktail (Bio-Rad Laboratories, Inc.). Total protein in each sample was measured using a bicinchoninic acid assay kit (Invitrogen; Thermo Fisher Scientific, Inc.), according to the manufacturer's protocol.Equal amounts of protein $(40 \mu \mathrm{g})$ were resolved using $10 \%$ SDS-PAGE gel electrophoresis and electro-transferred onto a PVDF membrane. Subsequently, the membrane was blocked for $60 \mathrm{~min}$ at room temperature with TBST containing 5\% skim milk. The membrane was incubated overnight at $4^{\circ} \mathrm{C}$ with the following primary antibodies: Anti-TLR2 (cat. no. ab16894; 1:1,000; Abcam), anti-IL-16 (cat. no. ab207181; 1:1,000; Abcam) and anti- $\beta$-actin (cat. no. ab115777; 1:1,000; Abcam). After washing with PBS, the membrane was incubated with horseradish peroxide-labeled secondary antibodies (cat. no. 7076s; 1:10,000; Cell Signaling Technology Inc.) for $1 \mathrm{~h}$ at $37^{\circ} \mathrm{C}$ and was subsequently developed using an ECL method with the ECL ${ }^{\text {TM }}$ Western Blotting Detection Reagents (Sigma-Aldrich; Merck KGaA). The membrane was visualized using a Gel Doc EZ Imager (Bio-Rad Laboratories, Inc.) and the protein bands were analyzed using ImageJ software (version 1.8.0; National Institutes of Health) using $\beta$-actin as the loading control.

ELISA. The collected clinical and cellular samples were centrifuged at $4^{\circ} \mathrm{C}$ for $10 \mathrm{~min}$ at $1509.3 \mathrm{x} \mathrm{g}$, and the supernatant was transferred into sterile centrifuge tubes. ELISA kits including the human TNF- $\alpha$ ELISA kit (cat. no. RAB1089; Sigma-Aldrich; Merck KGaA), NF- $\mathrm{B}$ p65 ELISA kit (cat. no. ab176648; Abcam), human IFN- $\gamma$ ELISA kit (cat. no. RAB0223; Sigma-Aldrich; Merck KGaA), human IL-6 ELISA kit (cat. no. RAB0306; Sigma-Aldrich; Merck KGaA) and human IL-10 ELISA kit (cat. no. RAB0244; Sigma-Aldrich; Merck KGaA) were used according to the manufacture's protocol. The samples were diluted and added into a microtiter plate precoated with anti-TNF- $\alpha$, anti-NF- $\kappa \mathrm{B}$, anti-IFN, anti-IL6 and anti-IL10 antibodies. After being incubated for $30 \mathrm{~min}$ at $37^{\circ} \mathrm{C}, 50 \mu \mathrm{l}$ of ELISA reactive solution was added to each well of the microplate and incubated for another $30 \mathrm{~min}$ at $37^{\circ} \mathrm{C}$. Subsequently, $50 \mu \mathrm{l}$ of chromogenic reagent A was added into each well, followed by the addition of $50 \mu 1$ of chromogenic reagent B. The microplate was then gently shaken for $30 \mathrm{sec}$, and was subsequently incubated in the dark for $15 \mathrm{~min}$ at $37^{\circ} \mathrm{C}$. After the microplate was removed from the incubation, the wells presented a blue color. If the signal intensity was too low, the incubation time was slightly extended by $30 \mathrm{~min}$. At the end of incubation, $50 \mu \mathrm{l}$ Alkaline Phosphatase stop solution (Sigma-Aldrich; Merck KGaA) was added into each well to stop the reaction, and the color in the wells turned to yellow. Within 15 min of reaction termination, the optical density (OD) value of each well was measured at a wavelength of $450 \mathrm{~nm}$. A standard curve was then drawn for each target substance using OD and concentration values. Each experiment was repeated $\geq 3$ times.

Statistical analysis. Data are presented as the mean \pm SD. The data were analyzed using SPSS software (version 21.0; IBM Corp.). Student's t-test was used for comparison between two groups. One-way ANOVA test was used for comparison between multiple groups, followed by Tukey's test. The survival rates of patients with $\mathrm{ICH}$ in different groups were compared using the Kaplan-Meier analysis. $\mathrm{P}<0.05$ was considered to indicate a statistically significant difference.

\section{Results}

Patients carrying the TT genotype are associated with the lowest survival rate. The present study recruited 182 subjects with ICH, genotyped as CC $(n=28)$, CT $(n=67)$ and TT $(n=87)$ according to the SNP rs41291957. Demographic and clinicopathological characteristics of all participants were presented in Table I. Kaplan-Meier survival curves were plotted for and are presented in Fig. 1. Upon the assessment of post-ICH mortality in patients followed-up for $\geq 6$ months after their discharge, it was found that patients carrying the TT genotype of SNP rs41291957 were associated with the lowest survival rate, while the patients carrying the $\mathrm{CC}$ genotype of SNP rs41291957 were associated with the highest survival rate.

TLR2 is a direct target gene of miR-143. To predict the regulatory relationship between $\mathrm{miR}-143$ and TLR2, a computational analysis was conducted, and TLR2 was identified as a candidate target gene of miR-143, and a possible binding site of miR-143 was found in the 3'UTR of TLR2 (Fig. 2A). The luciferase activity of THP-1 and HPASMC cells co-transfected with wild-type or mutant TLR2 mRNA in conjunction with miR-143 or miRNA controls was investigated. Relative luciferase activity of THP-1 cells 
Table I. Demographic and clinicopathological characteristics of patients with ICH.

\begin{tabular}{|c|c|c|c|}
\hline Clinical variable & $\mathrm{CC}+\mathrm{CT}(\mathrm{n}=95)$ & TT $(n=87)$ & P-value \\
\hline Age, years & $60.5 \pm 15.3$ & $61.1 \pm 16.2$ & 0.8215 \\
\hline Female $(\%)$ & $39(41.3)$ & $34(40.6)$ & 0.6325 \\
\hline Hypertension (\%) & $77(81.3)$ & $69(78.1)$ & 0.4425 \\
\hline History of stroke (\%) & $11(12.0)$ & $13(15.6)$ & 0.1254 \\
\hline History of ICH (\%) & $6(6.7)$ & $8(9.3)$ & 0.2571 \\
\hline Hematoma size, cc & $23.5 \pm 3.6$ & $25.8 \pm 6.5$ & 0.6251 \\
\hline Admission intraventricular hemorrhage (\%) & $20(22.0)$ & $27(31.3)$ & 0.8248 \\
\hline Admission hydrocephalus (\%) & $55(58.7)$ & $46(53.1)$ & 0.1364 \\
\hline Intraventricular hemorrhage score & $7.2 \pm 4.1$ & $7.8 \pm 6.6$ & 0.6481 \\
\hline Midline shift, mm & $2.3 \pm 0.8$ & $2.6 \pm 1.4$ & 0.1351 \\
\hline Hematoma evacuation $(\%)$ & $11(12.0)$ & $10(12.5)$ & 0.5124 \\
\hline Ventricular drainage $(\%)$ & $64(68.0)$ & $54(62.5)$ & 0.1354 \\
\hline Intrathecal tissue plasminogen activator (\%) & $5(5.3)$ & $5(6.3)$ & 0.8452 \\
\hline Ventricular shunt $(\%)$ & $6(6.0)$ & $8(9.3)$ & 0.2458 \\
\hline Pneumonia (\%) & $33(34.7)$ & $33(37.5)$ & 0.4518 \\
\hline Ventriculitis/meningitis (\%) & $3(3.3)$ & $3(3.1)$ & 0.8479 \\
\hline Sepsis $(\%)$ & $3(3.3)$ & $3(3.1)$ & 0.8479 \\
\hline
\end{tabular}

$\mathrm{ICH}$, intracerebral hemorrhage.

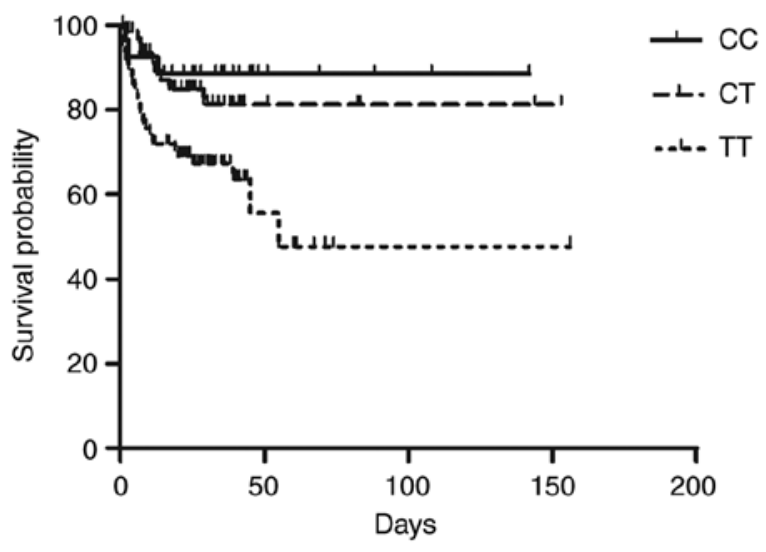

Figure 1. Patients carrying the TT genotype present the lowest survival rate. Kaplan-Meier survival curves were plotted for patients with ICH carrying the CC, CT and TT genotypes of rs41291957 single nucleotide polymorphism. $\mathrm{ICH}$, intracerebral hemorrhage.

co-transfected with wild-type TLR 2 mRNA and miR-143 was significantly downregulated compared with the THP-1 cells co-transfected with wild-type TLR2 mRNA and miRNA controls (Fig. 2B). However, cells transfected with mutant TLR2 mRNA exhibited a similar level of luciferase activity in the presence of miR-143 or miRNA controls (Fig. 2B). Similar results were obtained in HPASMC cells (Fig. 2C). The transfection of miR-143 mimics alone also significantly increased the expression level of miR-143 in THP-1 (Fig. 2D) and HPASMC (Fig. 2E) cells, indicating the successful transfection of miR-143 mimics. Collectively, the present results suggested that TLR2 mRNA was a target of miR-143.
IL-16 mRNA is not a target of miR-143 and are not associated. IL-16 is involved in post-ICH inflammation. The TargetScan online bioinformatics tool was used to locate the possible binding site of miR-143 in the 3'UTR of IL-16 mRNA (Fig. 3A). A luciferase assay was performed by co-transfecting THP-1 and HPASMC cells with wild-type or mutant IL-16 mRNA in conjunction with miR-143 or miRNA controls. No significant difference was observed between THP-1 cells co-transfected with wild-type or mutant IL-16 mRNA in conjunction with miR-143 or miRNA controls (Fig. 3B). Similar results were also obtained in HPASMC cells (Fig. 3C), indicating the absence of a regulatory relationship between miR-143 and IL-16 mRNA.

Effect of miR-143 overexpression on the expression levels of $I L-16$ and TLR2. The expression levels of IL-16 mRNA and TLR2 mRNA were measured in THP-1 and HPASMC cells transfected with miR-143 precursor, IL-16 siRNA or TLR2 siRNA. The relative expression of miR-143 in THP-1 cells was significantly increased in the presence of miR-143 precursor, demonstrating successful transfection of miR-143 precursor in THP-1 cells (Fig. 4A). The relative expression of IL-16 mRNA in THP-1 cells (Fig. 4B) was suppressed by IL-16 siRNA, while other treatments failed to affect the relative expression of IL-16. However, the relative expression of TLR2 mRNA was markedly reduced in the THP-1 cells (Fig. 4C) transfected with either miR-143 precursor or TLR2 siRNA. In addition, the expression levels of IL-16 and TLR2 proteins were altered in the presence of IL-16 siRNA and miR-143 precursor or TLR2 siRNA, respectively (Fig. 4D). Similar results were also obtained in HPASMC cells (Fig. 5), which further indicated the presence of a negative regulatory relationship between miR-143 and TLR2. 
A

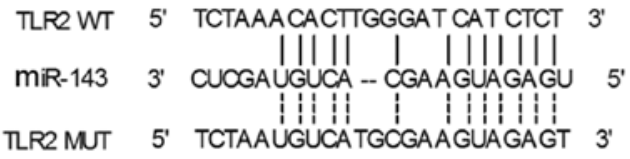

B

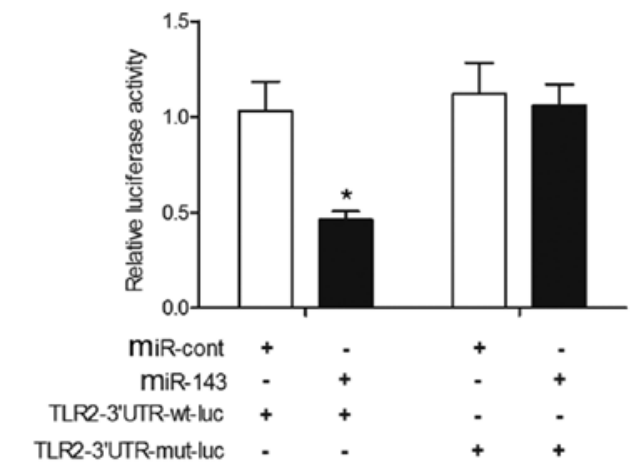

C

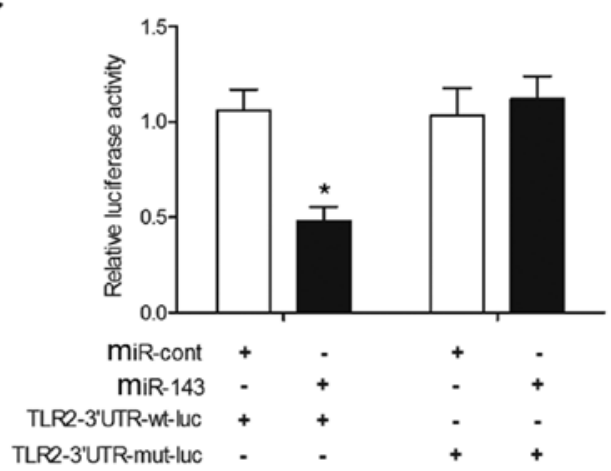

D

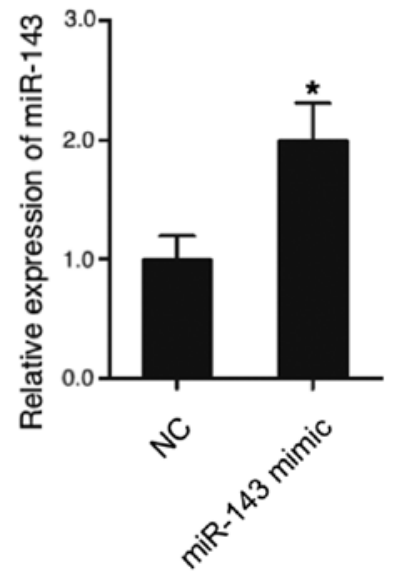

E

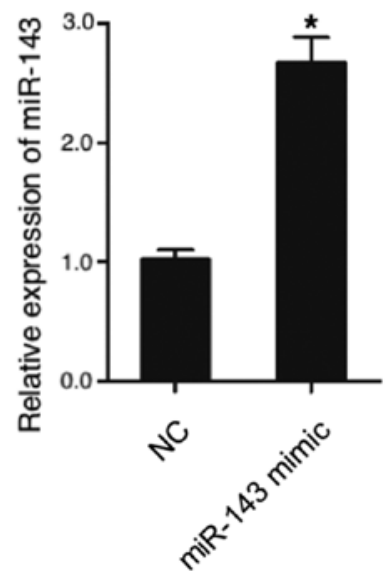

Figure 2. TLR2 is a direct target gene of miR-143. (A) Computational analysis of the regulatory relationship between miR-143 and TLR2 mRNA. (B) Luciferase assay of THP-1 cells co-transfected with wild-type or mutant TLR2 mRNA, and miR-143 or miRNA controls. (C) Luciferase assay of HPASMC cells co-transfected with wild-type or mutant TLR2 mRNA, and miR-143 or miRNA controls. (D) Relative expression of miR-143 in THP-1 cells transfected with miR-143 mimics. (E) Relative expression of miR-143 in HPASMC cells transfected with miR-143 mimics. $n=3$. ${ }^{*} \mathrm{P}<0.05$ vs. negative control group. TLR2, Toll-like receptor 2; miR-143, microRNA-143; miR-cont, microRNA-control; 3'-UTR, 3'-untranslated region; WT, wild-type; MUT, mutant; NC, negative control.

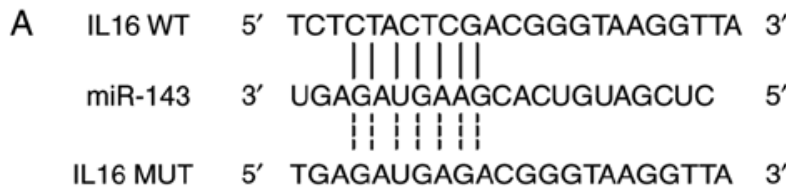

B

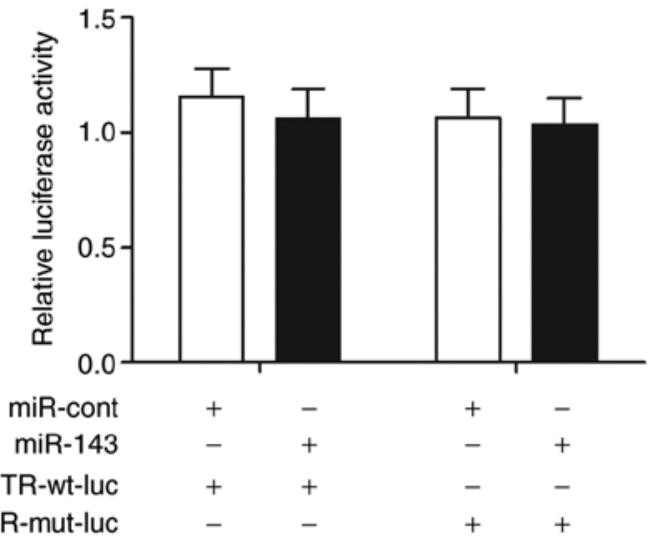

C

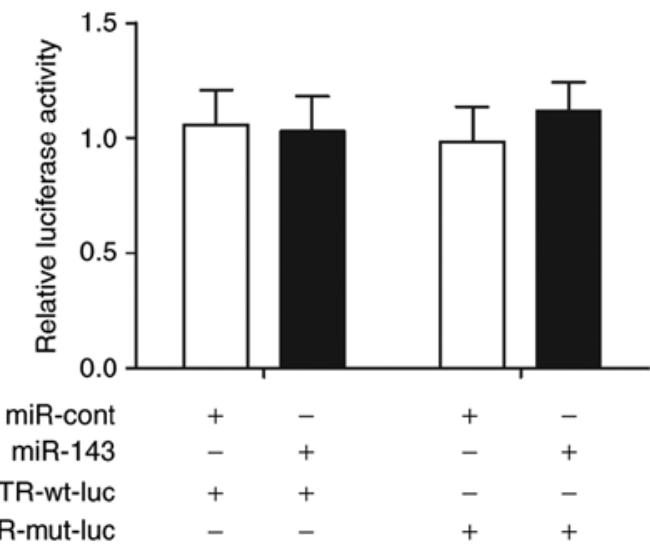

Figure 3. IL-16 mRNA is not a target of miR-143. (A) Computational analysis of the regulatory relationship between miR-143 and IL-16 mRNA. (B) Luciferase assay of THP-1 cells co-transfected with wild-type or mutant IL-16 mRNA, and miR-143 or miRNA controls. (C) Luciferase assay of HPASMC cells co-transfected with wild-type or mutant IL-16 mRNA, and miR-143 or miRNA controls. IL-16, interleukin-16; miR-143, microRNA-143; 3'-UTR, 3'-untranslated region; WT, wild-type; MUT, mutant.

Rs41291957 polymorphism affects the production of inflammatory factors in patients with ICH. The expression of other inflammatory factors, including TNFa, IFN, IL-6, IL-10 and $\mathrm{NF}-\kappa \mathrm{B}$, were compared in patients with $\mathrm{ICH}$ carrying different genotypes of rs41291957 SNP. The expression levels of TNF $\alpha$, IFN, IL-6, IL-10 and NF- $\kappa$ B in the CSF samples were highest in patients with $\mathrm{ICH}$ and the $\mathrm{CC}$ genotype, but were significantly reduced in patients with $\mathrm{CT}$ and TT genotypes (Fig. 6A-E). In contrast, the level of miR-143 in the CSF samples was lowest in patients with ICH and the CC genotype, while miR-143 levels were increased in patients with CT and TT genotypes (Fig. 6F). Similar results were also obtained in serum samples (Fig. 7), indicating that the SNP rs41291957 located in the promoter region of miR-143 could affect the transcription efficiency of miR-143. Therefore, the increased miR-143 expression in the patients carrying the TT genotype of SNP rs41291957 could increase the expression of pro-inflammatory factor TLR2, thus resulting in a poorer prognosis of $\mathrm{ICH}$. 
A

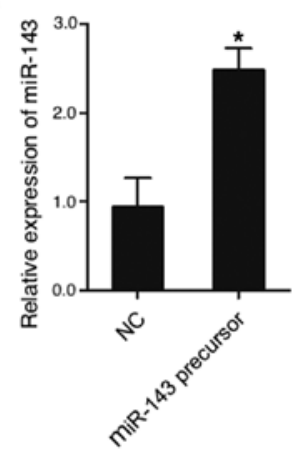

C

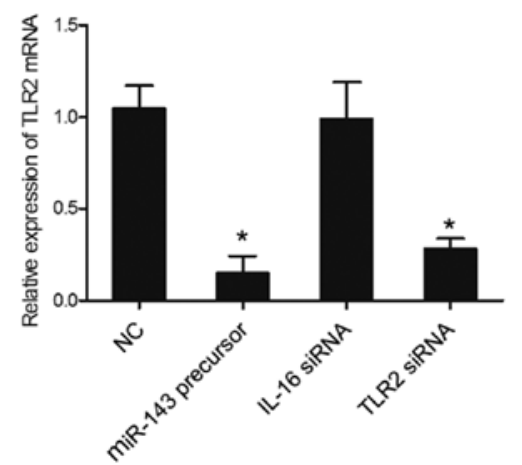

B

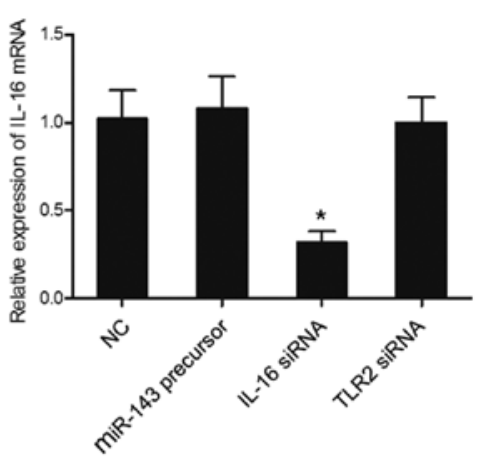

D

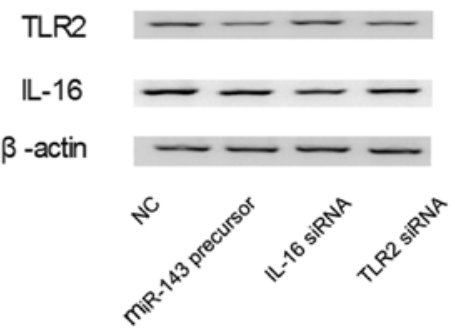

Figure 4. Expression levels of IL-16 mRNA and TLR2 mRNA in THP-1 cells. (A) Relative expression of miR-143 in THP-1 cells transfected with miR-143 precursor. (B) Relative expression of IL-16 mRNA in THP-1 cells transfected with miR-143 precursor, IL-16 siRNA or TLR2 siRNA. (C) Relative expression of TLR2 mRNA in THP-1 cells transfected with miR-143 precursor, IL-16 siRNA or TLR2 siRNA. (D) Western blot analysis results of IL-16 and TLR2 in THP-1 cells transfected with miR-143 precursor, IL-16 siRNA or TLR2 siRNA. $\mathrm{n}=3$. "P<0.05 vs. negative control group. IL-16, interleukin-16; miR-143, microRNA-143; TLR2, toll-like receptor 2; siRNA; small interfering RNA; NC, negative control.

A

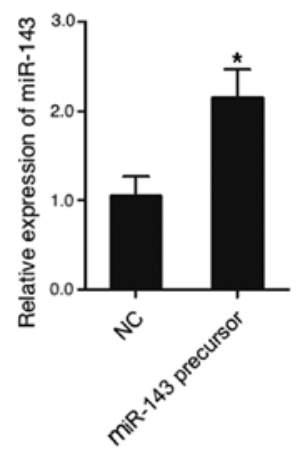

C

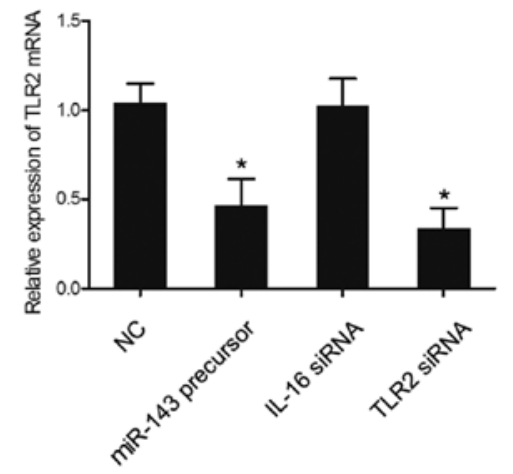

B

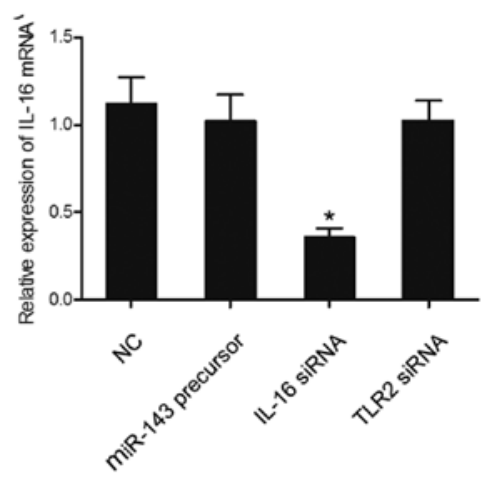

D

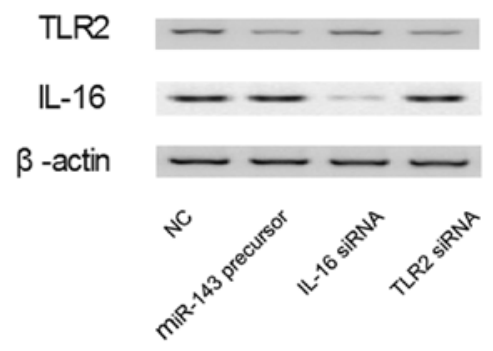

Figure 5. Expression levels of IL-16 mRNA and TLR2 mRNA in HPASMC cells. (A) Relative expression of miR-143 in HPASMC cells transfected with miR-143 precursor. (B) Relative expression of IL-16 mRNA in HPASMC cells transfected with miR-143 precursor, IL-16 siRNA or TLR2 siRNA. (C) Relative expression of TLR2 mRNA in HPASMC cells transfected with miR-143 precursor, IL-16 siRNA or TLR2 siRNA. (D) Western blot analysis results of IL-16 and TLR2 in HPASMC cells transfected with miR-143 precursor, IL-16 siRNA or TLR2 siRNA. $n=3$. " $\mathrm{P}<0.05$ vs. negative control group. IL-16, interleukin-16; miR-143, microRNA-143; TLR2, toll-like receptor 2; siRNA; small interfering RNA; NC, negative control. 

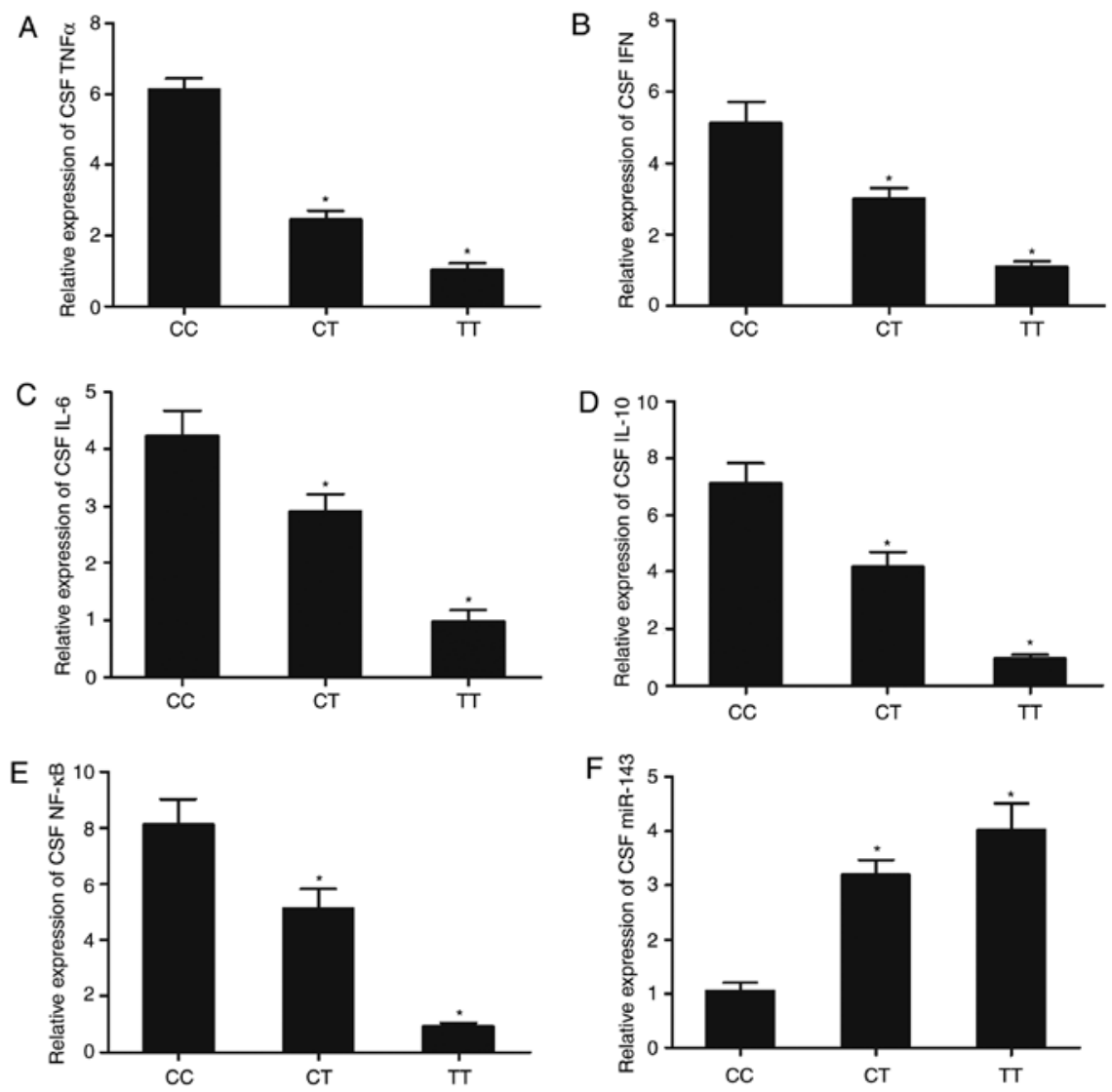

Figure 6. Expression of miR-143 and inflammatory factors, including TNF $\alpha$, IFN, IL-6, IL-10, and NF- $\mathrm{kB}$, in CSF samples genotyped as CC, CT and TT. Expression of (A) TNFa, (B) IFN, (C) IL-6, (D) IL-10, (E) NF- $\mathrm{kB}$ and (F) miR-143 in CSF samples from patients genotyped as CC, CT and TT. n=3. "P<0.05 vs. CC group. miR-143, microRNA-143; TNF $\alpha$, tumor necrosis factor $\alpha$; IFN, interferon; IL, interleukin; CSF, cerebrospinal fluid.
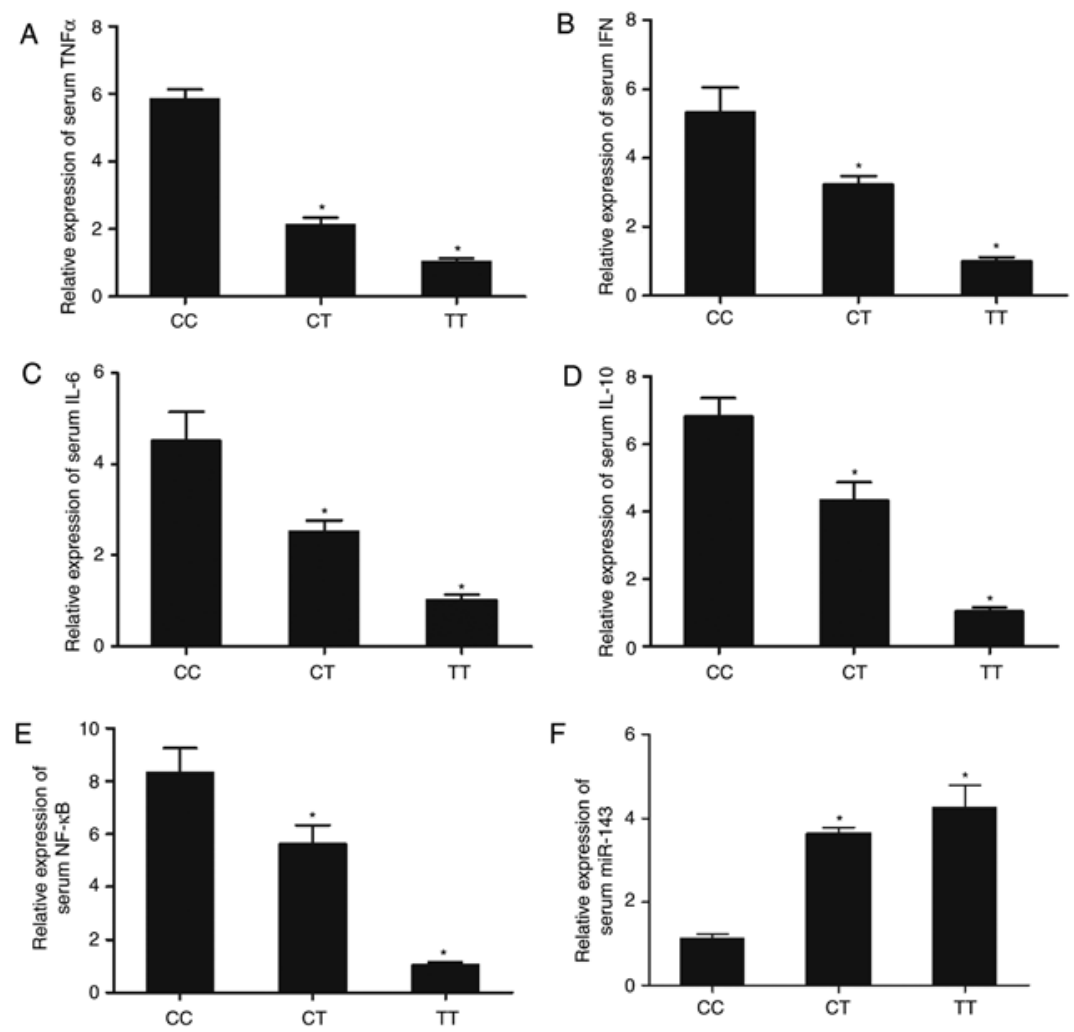

Figure 7. Expression of miR-143 and inflammatory factors, including TNF $\alpha$, IFN, IL-6, IL-10, and NF-kB, in serum samples genotyped as CC, CT and TT. Expression of (A) TNFa, (B) IFN, (C) IL-6, (D) IL-10, (E) NF-kB and (F) miR-143 in serum samples from patients genotyped as CC, CT and TT. n=3. ${ }^{*} \mathrm{P}<0.05$ vs. CC group. miR-143, microRNA-143; TNF $\alpha$, tumor necrosis factor $\alpha$; IFN, interferon; IL, interleukin; CSF, cerebrospinal fluid. 

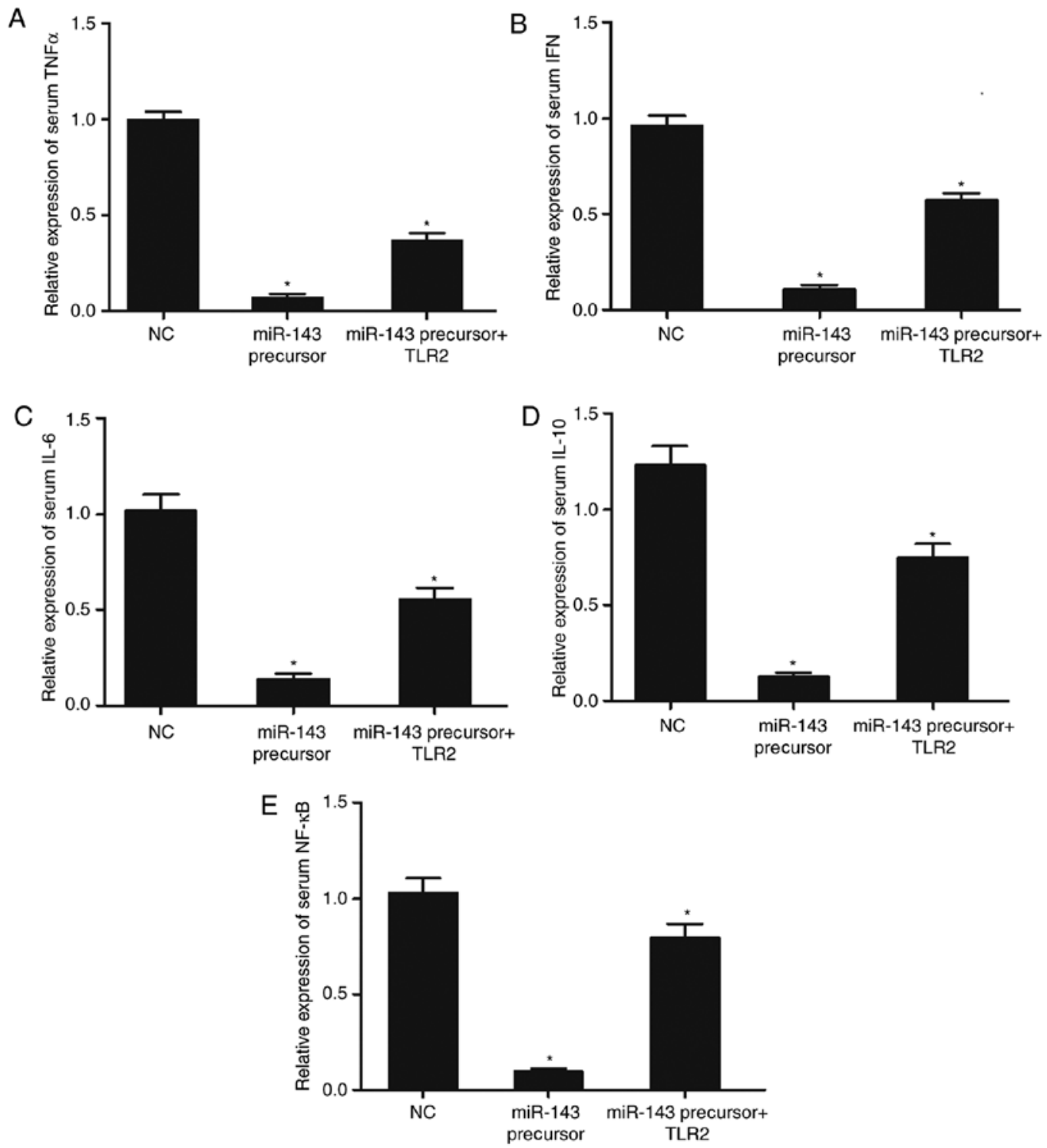

Figure 8. Effect of miR-143 transfection in the absence or presence of TLR2 overexpression on the production of inflammatory factors in THP-1 cells. (A) Transfection of miR-143 reduced the production of TNF $\alpha$, whereas the presence of TLR2 increased the production of TNF $\alpha$. (B) Transfection of miR-143 reduced the production of IFN, whereas the presence of TLR2 increased the production of IFN. (C) Transfection of miR-143 reduced the production of IL-6, whereas the overexpression of TLR2 increased the production of IL-6. (D) Transfection of miR-143 reduced the production of IL-10, whereas the presence of TLR2 increased the production of IL-10. (E) Transfection of miR-143 reduced the production of NF- $\mathrm{KB}$, whereas the overexpression of TLR2 increased the production of NF-kB. $n=3$. "P $<0.05$ vs. NC group. miR-143, microRNA-143; TLR2, toll-like receptor 2; TNF $\alpha$, tumor necrosis factor $\alpha$; IFN, interferon; IL, interleukin; CSF, cerebrospinal fluid; NC, negative control.

Effect of miR-143 transfection in the presence or absence of TLR2 overexpression on the production of inflammatory factors in THP-1 cells. Transfection of miR-143 precursors downregulated the production of TNF $\alpha$, IFN, IL-6, IL-10 and NF- $\mathrm{KB}$, while the overexpression of TLR2 promoted the production of these inflammatory cytokines (Fig. 8).

\section{Discussion}

$\mathrm{ICH}$ is a serious disease associated with high mortality (23). However, there are no effective treatments currently available to treat ICH (23). In addition, the chronic and acute damages caused by ICH, such as primary mechanical injuries in the brain tissues and secondary complications induced by inflammatory responses, further reduced the efficacy of ICH treatments (8). Therefore, an anti-inflammatory approach may be favorable for ICH treatment, by reducing inflammation-induced secondary brain damages, to improve the quality of life of patients (9). Previous studies have suggested that the inflammation of brain tissues not only affects post-ICH brain recovery, but also plays essential roles in the recovery from other brain traumas $(24,25)$. In addition, the pro-inflammatory factors secreted by endothelial cells, neuronal cells, astrocytes and microglia during brain injury can activate cellular adhesion molecules, thereby enhancing the recruitment of leukocytes into brain parenchyma and aggravating post-ICH brain injury $(5,24,25)$.

A previous study has shown that miR-143 can play a role in the prevention of ischemia-induced brain injury (26). The present study investigated the regulatory relationship 
between miR-143 and TLR2 via computational analysis and luciferase assay. TLR2 was identified as a target gene of miR-143, with a miR-143 binding site located in the 3'UTR of TLR2. In addition, the downregulated luciferase activity in the cells co-transfected with wild-type TLR2 mRNA and miR-143 suggested that TLR2 mRNA was a target of miR-143. Furthermore, no regulatory relationship was found between IL-16 and miR-143.

TLR2 is a transmembrane receptor with the ability to recognize peptidoglycan and lipoteichoic acid secreted by bacteria (27). In addition, TLR2 can induce the production of inflammatory signals (28). Previous studies have shown that TLR2 acts as a receptor for inflammatory factors, including high mobility group box-1, soluble CD14, hyaluronan and heat shock proteins, generated by pathogens and endogenously (27-32). In addition, several studies have hypothesized that the activation of TLR2 by these factors could be implicated in the generation of inflammatory responses in various of neurological diseases $(33,34)$. The present study measured the expression levels of IL-16 mRNA and TLR2 mRNA in the cells transfected with miR-143 precursor, IL-16 siRNA and TLR2 siRNA. The present results showed that the relative expression levels of TLR 2 mRNA was inhibited in the cells treated with miR-143 precursor or TLR2 siRNA. However, the relative expression of IL-16 mRNA was only reduced in the cells treated with IL-16 siRNA. The present results were also validated by western blot analysis. In a previous study, TLR2 inhibition in mice was shown to reduce post-ICH neutrophil infiltration. Neutrophil infiltration can induce tissue damage via the release and production of reactive oxygen species and proteases, thus affecting the induction of myeloperoxidase (MPO) activity after the onset of ICH (35). Furthermore, the oxidative stress caused by MPO can trigger neuronal apoptosis (36-38). Therefore, a reduction in neutrophil infiltration may consecutively attenuate the severity of ICH-induced brain injury. These previous studies demonstrated that the occurrence of post-ICH secondary brain injury is closely related to TLR2 activation as well as subsequent matrix metallopeptidase 9 stimulation and BBB disruption (35-38). The present study measured the expression of inflammatory factors, including TNF $\alpha$, IFN, IL-6, IL-10 and NF- $\kappa \mathrm{B}$, as well as the expression of miR-143 in the CSF/serum samples collected from patients with ICH. The expression of TNF $\alpha$, IFN, IL-6, IL-10 and $\mathrm{NF}-\kappa \mathrm{B}$ was increased in subjects carrying the CC genotype, and decreased in CT and TT genotypes of SNP rs41291957. Meanwhile, the level of miR-143 showed an opposite trend as that of above inflammatory factors.

In previous studies, miR-143 was shown to function as a tumor suppressor in the pathogenesis of a wide array of cancer types (17,39-42). Li et al (16) showed that the rs41291957 SNP in pri-miR-143 could alter the susceptibility to colorectal cancer. A previous study showed that, by targeting adducin3, miR-143 played an essential role in the morphogenesis of cardiac chamber in zebrafish (43). Miyasaka et al (44) demonstrated that cardiogenesis is mediated by miR-143. The present study examined Kaplan-Meier survival curves for 182 patients with ICH carrying CC, CT and TT genotypes of SNP rs41291957. The patients carrying the TT genotype were associated with the lowest survival rate, while the patients carrying the $\mathrm{CC}$ genotype were associated with the highest survival rate. rs41291957 SNP was found to be associated with the expression level of miR-143. Additionally, miR-143 was identified to target TLR2, whose expression could be associated with the severity of inflammatory conditions and the prognosis of ICH. Therefore, rs41291957 SNP may be used as a novel biomarker to predict the prognosis of $\mathrm{ICH}$.

\section{Acknowledgements}

Not applicable.

\section{Funding}

No funding was received.

\section{Availability of data and materials}

The datasets used and/or analyzed during the current study are available from the corresponding author on reasonable request.

\section{Authors' contributions}

$X Y$ and $X S$ designed the study. ZG and FC evaluated the literature. XY, ZG, FC, ZT and ZH collected and analyzed the data. XY and XS wrote the manuscript. All authors approved the final manuscript.

\section{Ethics approval and consent to participate}

The Human Research Ethics Committees of The First Affiliated Hospital of Chongqing Medical University provided approval for the present study. All methods were performed in accordance with the Declaration of Helsinki. Written informed consent was obtained from all patients or their first-degree relatives prior to surgery.

\section{Patient consent for publication}

Not applicable.

\section{Competing interests}

The authors declare that they have no competing interests.

\section{References}

1. Wang MD, Wang Y, Xia YP, Dai JW, Gao L, Wang SQ, Wang HJ, Mao L, Li M, Yu SM, et al: High serum miR-130a levels are associated with severe perihematomal edema and predict adverse outcome in acute ICH. Mol Neurobiol 53: 1310-1321, 2016.

2. Rincon F, Friedman DP, Bell R, Mayer SA and Bray PF: Targeted temperature management after intracerebral hemorrhage (TTM-ICH): Methodology of a prospective randomized clinical trial. Int J Stroke 9: 646-651, 2014.

3. Zhang Y, Chen Y, Wu J, Manaenko A, Yang P, Tang J, Fu W and Zhang JH: Activation of dopamine D2 receptor suppresses neuroinflammation through $\alpha \mathrm{B}$-crystalline by inhibition of $\mathrm{NF}-\kappa \mathrm{B}$ nuclear translocation in experimental ICH mice model. Stroke 46: 2637-2646, 2015.

4. Xu C, Wang T, Cheng S and Liu Y: Increased expression of T cell immunoglobulin and mucin domain 3 aggravates brain inflammation via regulation of the function of microglia/macrophages after intracerebral hemorrhage in mice. J Neuroinflammation 10: 141,2013 
5. Chen M, Li X, Zhang X, He X, Lai L, Liu Y, Zhu G, Li W, Li H, Fang Q, et al: The inhibitory effect of mesenchymal stem cell on blood-brain barrier disruption following intracerebral hemorrhage in rats: Contribution of TSG-6. J Neuroinflammation 12 $61,2015$.

6. Lei B, Dawson HN, Roulhac-Wilson B, Wang H, Laskowitz DT and James ML: Tumor necrosis factor $\alpha$ antagonism improves neurological recovery in murine intracerebral hemorrhage. J Neuroinflammation 10: 103, 2013.

7. Harder LM, Bunkenborg J and Andersen JS: Inducing autophagy: A comparative phosphoproteomic study of the cellular response to ammonia and rapamycin. Autophagy 10: 339-355, 2014.

8. Wakisaka Y, Chu Y, Miller JD, Rosenberg GA and Heistad DD: Spontaneous intracerebral hemorrhage during acute and chronic hypertension in mice. J Cereb Blood Flow Metab 30: 56-69, 2010

9. Rincon F and Mayer SA: Novel therapies for intracerebral hemorrhage. Curr Opin Crit Care 10: 94-100, 2004.

10. Bartel DP: MicroRNAs: Target recognition and regulatory functions. Cell 136: 215-233, 2009.

11. Nadal E, Truini A, Nakata A, Lin J, Reddy RM, Chang AC Ramnath N, Gotoh N, Beer DG and Chen G: A novel serum 4-microRNA signature for lung cancer detection. Sci Rep 5: 12464, 2015.

12. Akao Y, Nakagawa $Y$ and Naoe T: MicroRNAs 143 and 145 are possible common onco-microRNAs in human cancers. Oncol Rep 16: 845-850, 2006.

13. Starczynowski DT, Kuchenbauer F, Argiropoulos B, Sung S, Morin R, Muranyi A, Hirst M,Hogge D, Marra M, Wells RA, et al: Identification of miR-145 and miR-146a as mediators of the 5q-syndrome phenotype. Nat Med 16: 49-58, 2010.

14. Xie H, Lim B and Lodish HF: MicroRNAs induced during adipogenesis that accelerate fat cell development are downregulated in obesity. Diabetes 58: 1050-1057, 2009.

15. Cordes KR, Sheehy NT, White MP, Berry EC, Morton SU, Muth AN, Lee TH, Miano JM, Ivey KN and Srivastava D: miR-145 and miR-143 regulate smooth muscle cell fate and plasticity. Nature 460: 705-710, 2009.

16. Li L, Pan X, Li Z, Bai P, Jin H, Wang T, Song C, Zhang L and Gao L: Association between polymorphisms in the promoter region of miR-143/145 and risk of colorectal cancer. Hum Immunol 74: 993-997, 2013.

17. Wu D, Huang P, Wang L, Zhou Y, Pan H and Qu P: MicroRNA-143 inhibits cell migration and invasion by targeting matrix metalloproteinase 13 in prostate cancer. Mol Med Rep 8: 626-630, 2013

18. Yin X, Sun S, Zhao J, Yang J, Lei X, Xu C and Li K: Rs4705342 polymorphism is involved in the tumorigenesis of $\mathrm{HBV}$ positive $\mathrm{HCC}$ by altering the binding affinity of HBV induced NF- $\kappa \mathrm{B}$ with the promoter region of microRNA-143. J Cell Biochem 119: 5233-5242, 2018

19. Liu X, Gong J and Xu B: miR-143 down-regulates TLR 2 expression in hepatoma cells and inhibits hepatoma cell proliferation and invasion. Int J Clin Exp Pathol 8: 12738-12747, 2015.

20. Min H, Hong J, Cho IH, Jang YH, Lee H, Kim D, Yu SW, Lee S and Lee SJ: TLR2-induced astrocyte MMP9 activation compromises the blood brain barrier and exacerbates intracerebral hemorrhage in animal models. Mol Brain 8: 23, 2015.

21. Wang YC, Zhou Y, Fang H, Lin S, Wang PF, Xiong RP, Chen J, Xiong XY, Lv FL, Liang QL and Yang QW: Toll-like receptor 2/4 heterodimer mediates inflammatory injury in intracerebral hemorrhage. Ann Neurol 75: 876-889, 2014.

22. Livak KJ and Schmittgen TD: Analysis of relative gene expression data using real-time quantitative PCR and the 2(-Delta Delta $\mathrm{C}(\mathrm{T})$ ) method. Methods 25: 402-408, 2001.

23. Burns JD, Fisher JL and Cervantes-Arslanian AM: Recent advances in the acute management of intracerebral hemorrhage. Neurosurg Clin N Am 29: 263-272, 2018.

24. Bernales S, Schuck S and Walter P: ER-phagy: Selective autophagy of the endoplasmic reticulum. Autophagy 3: 285-287, 2007.

25. Bampton ET, Goemans CG, Niranjan D, Mizushima N and Tolkovsky AM: The dynamics of autophagy visualized in live cells: From autophagosome formation to fusion with endo/lysosomes. Autophagy 1: 23-36, 2005.
26. Bai Y, Zhang Y, Han B, Yang L, Chen X, Huang R, Wu F, Chao J, Liu P, Hu G, et al: Circular RNA DLGAP4 ameliorates ischemic stroke outcomes by targeting miR-143 to regulate endothelial-mesenchymal transition associated with blood-brain barrier integrity. J Neurosci 38: 32-50, 2018.

27. Park JS, Svetkauskaite D, He Q, Kim JY, Strassheim D, Ishizaka A and Abraham E: Involvement of toll-like receptors 2 and 4 in cellular activation by high mobility group box 1 protein. J Biol Chem 279: 7370-7377, 2004.

28. Huang QQ, Sobkoviak R, Jockheck-Clark AR, Shi B, Mandelin AM II, Tak PP, Haines GK III, Nicchitta CV and Pope RM: Heat shock protein 96 is elevated in rheumatoid arthritis and activates macrophages primarily via TLR2 signaling. J Immunol 182: 4965-4973, 2009.

29. Ohashi K, Burkart V, Flohé S and Kolb H: Cutting edge: Heat shock protein 60 is a putative endogenous ligand of the toll-like receptor-4 complex. J Immunol 164: 558-561, 2000.

30. Vabulas RM, Ahmad-Nejad P, Ghose S, Kirschning CJ, Issels RD and Wagner H: HSP70 as endogenous stimulus of the Toll/interleukin-1 receptor signal pathway. J Biol Chem 277: 15107-15112, 2002

31. Termeer C, Benedix F, Sleeman J, Fieber C, Voith U, Ahrens T, Miyake K, Freudenberg M, Galanos C and Simon JC: Oligosaccharides of hyaluronan activate dendritic cells via toll-like receptor 4. J Exp Med 195: 99-111, 2002.

32. Bsibsi M, Bajramovic JJ, Van Duijvenvoorden E, Persoon C, Ravid R, Van Noort JM and Vogt MH: Identification of soluble CD14 as an endogenous agonist for Toll-like receptor 2 on human astrocytes by genome-scale functional screening of glial cell derived proteins. Glia 55: 473-482, 2007.

33. Hanke ML and Kielian T: Toll-like receptors in health and disease in the brain: Mechanisms and therapeutic potential. Clin Sci (Lond) 121: 367-387, 2011.

34. Lehnardt S: Innate immunity and neuroinflammation in the CNS: The role of microglia in Toll-like receptor-mediated neuronal injury. Glia 58: 253-263, 2010.

35. Weiss SJ: Tissue destruction by neutrophils. N Engl J Med 320: 365-376, 1989

36. Albers DS and Beal MF: Mitochondrial dysfunction and oxidative stress in aging and neurodegenerative disease. J Neural Transm Suppl 59: 133-154, 2000.

37. Franklin JL: Redox regulation of the intrinsic pathway in neuronal apoptosis. Antioxid Redox Signal 14: 1437-1448, 2011.

38. Valencia A and Morán J: Reactive oxygen species induce different cell death mechanisms in cultured neurons. Free Radic Biol Med 36: 1112-1125, 2004.

39. Guo H, Chen Y, Hu X, Qian G, Ge S and Zhang J: The regulation of Toll-like receptor 2 by miR-143 suppresses the invasion and migration of a subset of human colorectal carcinoma cells. Mol Cancer 12: 77, 2013.

40. Ma Q, Jiang Q, Pu Q, Zhang X, Yang W, Wang Y, Ye S, Wu S, Zhong G, Ren J, et al: MicroRNA-143 inhibits migration and invasion of human non-small-cell lung cancer and its relative mechanism. Int J Biol Sci 9: 680-692, 2013.

41. Ni Y, Meng L, Wang L, Dong W, Shen H, Wang G, Liu Q and Du J: MicroRNA-143 functions as a tumor suppressor in human esophageal squamous cell carcinoma. Gene 517: 197-204, 2013.

42. Zhang Y, Wang Z, Chen M, Peng L, Wang X, Ma Q, Ma F and Jiang B: MicroRNA-143 targets MACC1 to inhibit cell invasion and migration in colorectal cancer. Mol Cancer 11: 23, 2012.

43. Deacon DC, Nevis KR, Cashman TJ, Zhou Y, Zhao L, Washko D, Guner-Ataman B, Burns CG and Burns CE: The miR-143-adducin3 pathway is essential for cardiac chamber morphogenesis. Development 137: 1887-1896, 2010.

44. Miyasaka KY, Kida YS, Banjo T, Ueki Y, Nagayama K, Matsumoto T, Sato M and Ogura T: Heartbeat regulates cardiogenesis by suppressing retinoic acid signaling via expression of miR-143. Mech Dev 128: 18-28, 2011.

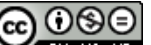

This work is licensed under a Creative Commons Attribution-NonCommercial-NoDerivatives 4.0 International (CC BY-NC-ND 4.0) License. 